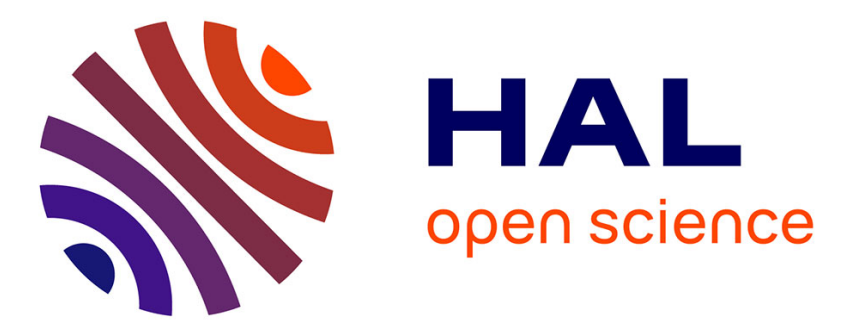

\title{
A multi-scale approach for the optimum design of sandwich plates with honeycomb core. Part I: homogenisation of core properties
}

\author{
Anita Catapano, Marco Montemurro
}

\section{- To cite this version:}

Anita Catapano, Marco Montemurro. A multi-scale approach for the optimum design of sandwich plates with honeycomb core. Part I: homogenisation of core properties. Composite Structures, 2014, 13p. 10.1016/j.compstruct.2014.07.057 . hal-01063337v2

\section{HAL Id: hal-01063337 \\ https://hal.science/hal-01063337v2}

Submitted on 12 Sep 2014

HAL is a multi-disciplinary open access archive for the deposit and dissemination of scientific research documents, whether they are published or not. The documents may come from teaching and research institutions in France or abroad, or from public or private research centers.
L'archive ouverte pluridisciplinaire HAL, est destinée au dépôt et à la diffusion de documents scientifiques de niveau recherche, publiés ou non, émanant des établissements d'enseignement et de recherche français ou étrangers, des laboratoires publics ou privés. 


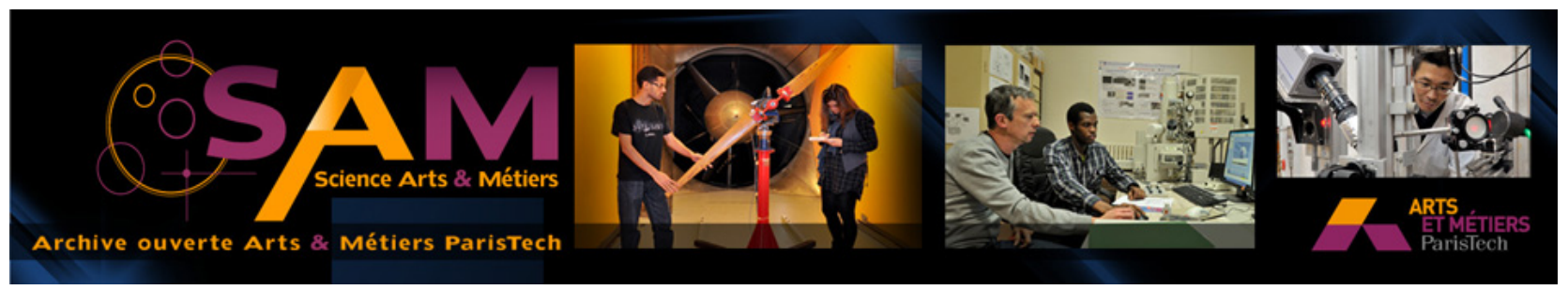

Science Arts \& Métiers (SAM)

is an open access repository that collects the work of Arts et Métiers ParisTech researchers and makes it freely available over the web where possible.

This is an author-deposited version published in: http://sam.ensam.eu

Handle ID: .http://hdl.handle.net/10985/8498

\section{To cite this version :}

Anita CATAPANO, Marco MONTEMURRO - A multi-scale approach for the optimum design of sandwich plates with honeycomb core. Part l: homogenisation of core properties - Composite Structures p.13p. - 2014 


\title{
A multi-scale approach for the optimum design of sandwich plates with honeycomb core. Part I: homogenisation of core properties
}

\author{
Anita Catapano \\ Université de Bordeaux, I2M CNRS UMR 5295, F-33400 Talence, France \\ Marco Montemurro* \\ Arts et Métiers ParisTech, I2M CNRS UMR 5295, F-33400 Talence, France.
}

Author for Correspondence:

Marco Montemurro, $\mathrm{PhD}$,

Arts et Métiers ParisTech,

I2M CNRS UMR 5295,

F-33400 Talence, France,

tel: +33556845422 ,

fax: +33540006964 ,

e.mail:marco.montemurro@ensam.eu,

marco.montemurro@u-bordeaux1.fr

*Corresponding author. 


\begin{abstract}
This work deals with the problem of the optimum design of a sandwich panel. The design process is based on a general two-level optimisation strategy involving different scales: the meso-scale for both the unit cell of the core and the constitutive layer of the laminated skins and the macro scale for the whole panel. Concerning the meso-scale of the honeycomb core, an appropriate model of the unit cell able to properly provide its effective elastic properties (to be used at the macro-scale) must be conceived. To this purpose, in this first paper, we present the numerical homogenisation technique as well as the related finite element model of the unit cell which makes use of solid elements instead of the usual shell ones. A numerical study to determine the effective properties of the honeycomb along with a comparison with existing models and a sensitive analysis in terms of the geometric parameters of the unit cell have been conducted. Numerical results show that shell-based models are no longer adapted to evaluate the core properties, mostly in the context of an optimisation procedure where the parameters of the unit cell can get values that go beyond the limits imposed by a $2 \mathrm{D}$ model.
\end{abstract}

\title{
Keywords:
}

Sandwich Structures; Honeycomb; Homogenisation; Composite materials.

\section{Notations}

GA Genetic Algorithm

\section{FE Finite Element}

$t_{c}$ Thickness of the aluminium foil used to produce the honeycomb core

$l_{1}$ Length of the oblique sides of the hexagonal repetitive unit cell

$l_{2}$ Length of the horizontal sides of the hexagonal repetitive unit cell

$\vartheta$ Corrugation angle of the hexagonal unit cell

$h_{c}$ Height of the honeycomb core

RVE Representative Volume Element for the honeycomb core

$V_{R V E}$ Volume of the representative volume element (including the second phase, i.e. the "elastic air")

$\left\{O ; x_{1}, x_{2}, x_{3}\right\}$ Global material frame of the representative volume element

$a_{1}$ Side length, along $x_{1}$-axis, of the representative volume element

$a_{2}$ Side length, along $x_{2}$-axis, of the representative volume element

$a_{3}$ Side length, along $x_{3}$-axis, of the representative volume element 
$V_{E F F}$ Effective volume of the unit cell of the honeycomb core

DOFs Degrees Of Freedom

$\bar{\sigma}_{\alpha}$ Volume averaged stress tensor components (Voigt's notation)

$\bar{\varepsilon}_{\beta}$ Volume averaged strain tensor components (Voigt's notation)

$C_{\alpha \beta}$ Stiffness tensor components of the homogenised core (Voigt's notation)

$\bar{\sigma}_{\alpha \beta}$ Volume averaged stress tensor components (tensorial notation)

$\bar{\varepsilon}_{\alpha \beta}$ Volume averaged strain tensor components (tensorial notation)

BCs Boundary Conditions

$u$ Arbitrarily imposed displacement

$E_{i}$ Young's moduli in the material frame $\left\{O ; x_{1}, x_{2}, x_{3}\right\}$

$G_{i j}$ Shear moduli in the material frame $\left\{O ; x_{1}, x_{2}, x_{3}\right\}$

$\nu_{i j}$ Poisson's ratios in the material frame $\left\{O ; x_{1}, x_{2}, x_{3}\right\}$

$\rho_{R V E}$ Effective (or relative) density of the honeycomb core

$\rho$ Density of the aluminium

$E$ Young's modulus of the aluminium

$\nu$ Poisson's ratio of the aluminium

$E_{a i r}$ Young's modulus of the elastic air

$\nu_{\text {air }}$ Poisson's ratio of the elastic air

$\{O ; \xi, \eta, z\}$ Local frame defined on the oblique side of the hexagonal cell

$\sigma_{\xi \xi}, \sigma_{\eta \eta}, \sigma_{\eta \xi}$ Local stress tensor components expressed within the local frame

LB Lower Bound

UB Upper Bound 


\section{Introduction}

Sandwich structures are widely used in several fields: aviation, automotive, naval, construction industry. Their application, in fact, ranges from the most performing structures such as aircraft wings, helicopters rotor blades, racing yachts keels to home furnishings. This is because sandwich structures offer high capacity of stiffness with a very low weight. The difference between a monolithic or laminated plate and a sandwich one concerns the presence of a low density cellular solid, i.e. the core, between two stiffer thin plates, that increases the geometric moment of inertia of the plate with a few weight increment.

However, when the choice of the designer falls on the use of a sandwich structure, for a given application, he must additionally define the type of sandwich structure that has to be employed. We can, in fact, identify several types of sandwich structures according to the geometry and shape of the core: honeycomb, solid, foam, corrugated, truss, web cores.

The most important feature of the core is its relative density (ratio between the density of the cellular material and that of the material from which the cells walls are made) that can vary from 0.001 to, generally, 0.4, see [15]. Almost any material can be used to build a cellular solid: polymers, metals, ceramics, composites, etc..

In aircraft and spacecraft applications sandwich panels are composed by glass or carbonfiber composite skins separated by aluminium or resin honeycombs, or by polymer foams. In particular, the honeycomb cell size can be chosen to provide cores with different stiffness and density properties. The result is a panel with very high bending stiffness-to-weight and strength-to-weight ratios. A review on sandwich structures and their applications can be found in $[15,27,29]$.

The optimal design of sandwich structures is much more cumbersome than that of a classical monolithic structure. The difficulties increase when the sandwich structure is made of composite skins and a honeycomb core. In this case we have to face, into the same design process, both the difficulty of designing a laminated plate (concerning the skins) and the difficulty of designing a complex 3D cellular core. Therefore, engineers always use some simplifying assumptions or rules to obtain, in an easier and faster way, a solution, e.g. the use of symmetric balanced stacks for the skins to ensure the membrane/bending elastic uncoupling along with the membrane orthotropy or the use of regular hexagonal geometry for the unit cell of the core. Such assumptions extremely shrink the solution space of potential configurations for the problem at hand. An alternative approach could consist in formulating the design problem of the sandwich panel as a constrained optimisation problem without introducing any simplifying hypothesis nor on the nature of the skins laminate neither on the geometry of the repetitive unit cell of the honeycomb core. Obviously, this can be done on one hand by a rigorous mathematical formulation of the problem and on the other hand by the use of numerical techniques that must be able to 
explore the whole design space in order to find the true global optimal configuration of the system. Moreover, unlike what is usually done in literature, our objective is twofold: on one hand we want to formulate and solve such a problem on different scales and on the other hand we want to include within the design process all possible parameters defining the structure (at each scale) as optimisation variables.

Therefore, in the framework of the design of a sandwich plate with honeycomb core and composite skins, the optimisation variables will be the material and the geometrical properties characterising both the skins and the core at each scale.

The design strategy proposed in this work is a numerical optimisation procedure that does not make any simplifying assumption in order to obtain a true global optimum configuration of the system. The design process is not submitted to restrictions, i.e. any parameter characterising our structure is an optimisation variable: thickness of the core, number of plies of skins, plies orientations and geometric parameters of the unit cell.

In order to face the design of the sandwich structure at both meso and macro scales, we used an optimisation strategy developed on two different levels: at the first level we determine the optimal geometry of the unit cell together with the material and geometric parameters of the laminated skins (at this level the laminate representing each skin is modelled as an equivalent homogeneous anisotropic plate whose behaviour at the macroscale is described in terms of tensor invariants, i.e. the laminate polar parameters, see [26]). At the second level of the strategy we determine the optimal skins lay-up (the skin meso-scale) meeting the values of the material and geometrical parameters issued from the first level of the strategy. The whole strategy is based on the use of the polar formalism $[26,28]$ and on the Genetic Algorithm (GA) BIANCA [20, 21]. For more details on the polar formalism and the related advantages the reader is addressed to Part II of the present work.

Concerning the model of the core, the first level of the strategy involves two different scales:

- the meso-scale wherein the core is modelled via the repetitive unit cell characterised by some geometric variables;

- the macro-scale where the core is modelled as an homogeneous orthotropic solid whose mechanical response is described through the full set of elastic moduli whose values depend on the geometric parameters of the unit cell.

Therefore, the link between these two scales is represented by the homogenisation phase of the honeycomb core. To this purpose we decided to develop an accurate method to determine the material properties of the orthotropic core that will be assigned to the equivalent solid at the macro-scale. 
A considerable number of analytical, numerical and experimental methods for predicting the effective elastic properties of the orthotropic honeycomb core as function of geometric and material characteristics of its unit cell can be found in the literature. The work of Gibson and Ashby [15] represents the first research study where the full set of elastic properties of a honeycomb core with constant wall thickness has been determined. Subsequently, this technique has been modified by Zhang and Ashby [30] to include the double thickness walls to determine the out-of-plane properties. Another analytical approach is presented in [19] using the laminate theory. In 1997, Burton and Noor [11] present the full set of nine material properties derived analytically considering the influence of the double thickness walls. Despite this last work represents a great step forward in the evaluation of the honeycomb structure properties it shows also a weakness: the out-of-plane shear properties of the honeycomb core are not univocally determined since the authors give only the lower and upper bounds for these properties. Other analytical approaches have been developed during the following years, for example [14] where the authors take into account also the skins effect or [23] where some experimental results are used to develop an analytical method to determine the out-of-plane mechanical properties. Other researchers developed different numerical approaches based on the Finite Element (FE) technique to investigate the material properties of honeycomb structures. Grediac [16] was one of the firsts to determine the out-of-plane properties of the honeycomb through a FE approach. An analogous work, were the transverse shear stiffness properties of honeycombs are determined through a FE method is presented in [24]. In [10] a displacement-based homogenisation technique is presented to evaluate the mechanical properties of foam-filled honeycomb cores. We can also find other works where the effective properties of the sandwich plate are determined via FE static analyses conducted on the overall geometry of the honeycomb core instead of performing an homogenisation process on the unit cell, see for instance [22]. Further interesting works on purely experimental or mixed numerical/experimental techniques for the determination of the effective properties of the honeycomb core can be found in $[13,18]$.

A common weakness of the works about FE-based homogenisation techniques consists in the use of shell-like models for the unit cell of the honeycomb core. These models do not take into account the true geometry of the cell and, consequently, they are not able to properly estimate the influence of the real 3D stress state on the effective core properties. In particular, in this work, we need an adequate model of the honeycomb core, at the meso-scale, able to provide with a good level of accuracy the material properties of the core for any combination of design variables that (in the framework of the optimisation procedure) could give rise to a geometry of the unit cell with thick walls rather than thin ones. Therefore, before introducing the design problem of the sandwich structure and the related two-level optimisation strategy, we decided to develop an accurate 3D model, for the numerical homogenisation phase of the core, that has to be included within the first 
level of the procedure and that represents the link between the meso and the macro-scale of the core.

In particular, concerning the evaluation of the effective material properties of the core, the skins effect, or skins influence, is not taken into account during the homogenisation phase. In this work we adopt a conventional approach where the sandwich plate is modelled, at the macro-scale level, as a heterogeneous continuum composed by the union of three parts:

- two laminated skins (that a priori can be different) whose anisotropic mechanical behaviour is described through a set of tensor invariants concerning both membrane, bending and coupling stiffness tensors (see Part II). Each skin is, hence, characterised by design variables of different nature (geometrical and material), i.e. its thickness (so the number of plies of the laminate) and its homogenised stiffness tensors invariants (namely the laminate polar parameters, see Part II);

- the core whose mechanical response (at the macro-scale) is defined by the elastic moduli of the equivalent continuum that uniquely depend upon the geometrical design variables of the unit cell (defined at the meso-scale).

Neglecting the interaction between the skins and the core, at the meso-scale level of the core, corresponds to adopt the so-called "free modulus model" in the framework of the determination of the effective core properties, see [10, 14].

Being this work rather long and considering the fact that it involves two different but linked main topics (the core homogenisation and the optimum design of the sandwich panel) we decided to divide its presentation into two parts. In this first part, we will present the numerical homogenisation technique as well as the 3D FE model used to deal with the core homogenisation problem. In part II, we will present the formulation of the optimisation problem along with the two-level strategy and some numerical examples to prove its effectiveness.

The paper is organised as follows: the description of the core homogenisation problem is introduced in Section 2 and the FE model used for the numerical homogenisation is presented in Section 3. In Section 4 we present a numerical study to determine the effective in-plane and out-of-plane properties of the honeycomb along with a comparison with the existing analytical and numerical models and a sensitive analysis in terms of the geometric parameters of the unit cell. Finally, Section 5 ends the paper with some concluding remarks.

\section{Homogenisation of core properties: problem description}

In the last decades, several analytical, numerical and experimental techniques have been developed in order to determine the effective properties of honeycomb sandwich cores as 
function of geometric and material properties of the repetitive unit cell. Each method presents a certain level of sophistication. For example, analytical techniques, based on energy methods or homogenisation methods, make use of some simplifying assumptions to obtain the elasticity solution of the unit cell. To avoid the use of such assumptions, several studies were conducted to develop new experimental-based and numerical-based techniques for determining the effective core properties.

On one hand, experimental-based methods, see for instance [3-6], require a standardised procedure for the measurements. However, the main drawbacks of these procedures consist in the fact that they are very expensive in terms of both time and money and the obtained results are valid only for the particular material and geometry of the sample employed in the analysis.

On the other hand, numerical-based techniques, such as FE methods, do not make use of the simplifying assumptions used in analytical approaches and are not expensive. In addition, they can lead to realistic solutions of the elasticity problem in terms of stress and strain fields over the unit cell or within the whole structure of the honeycomb core.

As a consequence, in this work we have chosen a FE-based approach as a numerical homogenisation technique to determine the core properties. It should be noted that a special feature of the honeycomb is its repetitiveness, i.e. the periodicity in its shape. Therefore, at this stage, we will apply the homogenisation method at the meso-scale (the scale of the unit cell) and then, we will replace the actual cellular structure, at the macro-scale level, by an equivalent homogeneous anisotropic medium characterised by the elastic properties determined during the homogenisation phase. The proposed FE-based homogenisation technique leads us to include all the geometric parameters of the unit cell among the optimisation variables of the process without a great loss of computational time (about 11 seconds on a $2.50 \mathrm{GHz}$ Dual Core processor for a single homogenisation analysis).

The basic assumptions made to evaluate the elastic response of our model and, hence, to determine the effective core properties are:

- linear, elastic behaviour for the material of the cell walls;

- perfect bonding for the wall-to-wall contact;

- the buckling of the cell walls is disregarded.

We recall that, as previously stated, since the aim of this first paper does not consist in developing an equivalent homogeneous plate/solid model for the whole sandwich panel, we do not consider the skins influence on the evaluation of the effective properties of the honeycomb core.

A scheme of the repetitive unit cell, used for the numerical homogenisation of the honeycomb core, is showed in Fig. 1. 


\section{A 3D finite element model for numerical homogenisation}

The effective properties of the core are determined using the strain energy homogenisation technique of periodic media, see [7]. This technique makes use of the repetitive unit of the periodic structure to approximate its effective properties at the macro-scale level. The basic feature of the strain energy homogenisation technique consists in the assumption that the repetitive unit of the periodic structure and the corresponding unit volume of the homogeneous solid undergo the same deformation having, hence, the same strain energy.

In this case, the periodic structure is the honeycomb core whose repetitive unit cell has three planes of symmetry, thus we decided to exploit these symmetries using, in the homogenisation process, only an eighth of the repetitive unit cell as shown in Fig. 2. The geometric characteristics of the repetitive unit used for the static analysis of the homogenisation model are shown in Fig. 3: $t_{c}$ is the thickness of the foil used to produce the honeycomb, $l_{1}$ is the length of the oblique side of the cell, $l_{2}$ represents the length of the horizontal sides of the repetitive unit while $\vartheta$ is the cell corrugation angle and $h_{c}$ is the height of the honeycomb core. We can now define the related Representative Volume Element (RVE) of the honeycomb core whose volume is (see Fig. 2 and 3):

$$
V_{\mathrm{RVE}}=a_{1} a_{2} a_{3}
$$

with:

$$
\begin{aligned}
& a_{1}=2 l_{2}+t_{c} \tan \left(\frac{\vartheta}{2}\right)+\left[l_{1}+t_{c} \tan \left(\frac{\vartheta}{2}\right)\right] \cos (\vartheta), \\
& a_{2}=\left[l_{1}+t_{c} \tan \left(\frac{\vartheta}{2}\right)\right] \sin (\vartheta)+t_{c} \\
& a_{3}=\frac{h_{c}}{2},
\end{aligned}
$$

whereas the effective volume of the unit cell is:

$$
V_{\mathrm{EFF}}=t_{c} \frac{h_{c}}{2}\left[l_{1}+2 l_{2}+2 t_{c} \tan \left(\frac{\theta}{2}\right)\right] .
$$

The FE model for the homogenisation process has been created within the commercial FE code ANSYS ${ }^{\circledR}$. We used the 20-node solid element SOLID186 with three Degrees Of Freedom (DOFs) per node. The model along its structured mesh is illustrated in Fig. 4. In this model the elements that do not belong to the unit cell geometry represent the "second phase" which has the properties of the so-called "elastic air" [2]. The second phase is introduced as a "numerical artifice" to obtain the strain field provided by the theory underlying the strain energy homogenisation technique, see [7] for more details. Unlike what is usually done in the literature, where the cell geometry is modelled with shell elements that show a geometric overlapping between the volumes built over the horizontal and oblique wall sides, in this work a 3D solid model is used to correctly represent the 
real geometry of the repetitive unit of the honeycomb structure. Our choice is due to the fact that, thanks to the 3D solid model, we can also take into account the effect of the full three-dimensional stress field in the determination of the effective core properties. As we will show in the numerical study of Section 4, this effect cannot be neglected in any case, mostly when the geometrical configuration of the unit cell is far away from a classical configuration of regular hexagon with thin walls.

A further assumption, as specified in Section 2, concerns the elastic behaviour of the orthotropic homogeneous medium. The generalised Hooke's law for the RVE can be written as follows:

$$
\left\{\begin{array}{c}
\bar{\sigma}_{1} \\
\bar{\sigma}_{2} \\
\bar{\sigma}_{3} \\
\bar{\sigma}_{4} \\
\bar{\sigma}_{5} \\
\bar{\sigma}_{6}
\end{array}\right\}=\left[\begin{array}{cccccc}
C_{11} & C_{12} & C_{13} & 0 & 0 & 0 \\
C_{21} & C_{22} & C_{23} & 0 & 0 & 0 \\
C_{31} & C_{32} & C_{33} & 0 & 0 & 0 \\
0 & 0 & 0 & C_{44} & 0 & 0 \\
0 & 0 & 0 & 0 & C_{55} & 0 \\
0 & 0 & 0 & 0 & 0 & C_{66}
\end{array}\right]\left\{\begin{array}{l}
\bar{\varepsilon}_{1} \\
\bar{\varepsilon}_{2} \\
\bar{\varepsilon}_{3} \\
\bar{\varepsilon}_{4} \\
\bar{\varepsilon}_{5} \\
\bar{\varepsilon}_{6}
\end{array}\right\},
$$

where $\bar{\sigma}$ and $\bar{\varepsilon}$ are, respectively, the stress and strain tensors expressed in Voigt's notation. The link between tensorial and Voigt's notation for both tensors is:

$$
\left\{\begin{array}{l}
\bar{\sigma}_{1} \\
\bar{\sigma}_{2} \\
\bar{\sigma}_{3} \\
\bar{\sigma}_{4} \\
\bar{\sigma}_{5} \\
\bar{\sigma}_{6}
\end{array}\right\}=\left\{\begin{array}{l}
\bar{\sigma}_{11} \\
\bar{\sigma}_{22} \\
\bar{\sigma}_{33} \\
\bar{\sigma}_{23} \\
\bar{\sigma}_{13} \\
\bar{\sigma}_{12}
\end{array}\right\}, \quad\left\{\begin{array}{l}
\bar{\varepsilon}_{1} \\
\bar{\varepsilon}_{2} \\
\bar{\varepsilon}_{3} \\
\bar{\varepsilon}_{4} \\
\bar{\varepsilon}_{5} \\
\bar{\varepsilon}_{6}
\end{array}\right\}=\left\{\begin{array}{c}
\bar{\varepsilon}_{11} \\
\bar{\varepsilon}_{22} \\
\bar{\varepsilon}_{33} \\
2 \bar{\varepsilon}_{23} \\
2 \bar{\varepsilon}_{13} \\
2 \bar{\varepsilon}_{12}
\end{array}\right\} .
$$

As $\bar{\sigma}$ and $\bar{\varepsilon}$ are referred to the equivalent homogeneous solid, each component of $\bar{\sigma}$ and $\bar{\varepsilon}$ is volume averaged.

The main objective of our homogenisation process is to determine all non-zero components of the stiffness tensor $\mathbf{C}$ of Eq. (4). The expression of the components of $\mathbf{C}$ in terms of those of the stress and strain tensors is:

$$
C_{\alpha \beta}=\frac{\bar{\sigma}_{\alpha}}{\bar{\varepsilon}_{\beta}} \text { with } \alpha, \beta=1,2, \cdots, 6 \text { and } \varepsilon_{\gamma}=0 \text { with } \gamma=1,2, \cdots, 6, \quad \gamma \neq \beta .
$$

We have to determine, now, the nine independent components of $\mathbf{C}$.

In [7] the strain energy homogenisation technique is applied to the calculation of the elastic moduli of unidirectional laminae. In this work the author determines the five components of the transversally isotropic ply stiffness tensor by solving four different static analyses for the FE model of the RVE. In each one of these analyses the boundary conditions (BCs) are imposed in order to obtain a strain tensor having only one component different from zero. According to [7] we have determined the nine independent components of $\mathbf{C}$ for the honeycomb core through six static analyses on the FE model of Fig. 4. The corresponding BCs for each static analysis are resumed in Tables 1 and 2. 
These BCs are imposed in order to satisfy the symmetries of the RVE and to generate a strain field in such a way that only one component of the tensor $\bar{\varepsilon}$ is different from zero for each static analysis. The relations giving the volume average strain components are:

$$
\bar{\varepsilon}_{1}=\frac{u}{a_{1}}, \bar{\varepsilon}_{2}=\frac{u}{a_{2}}, \bar{\varepsilon}_{3}=\frac{u}{a_{3}}, \bar{\varepsilon}_{4}=\frac{u}{a_{3}}, \bar{\varepsilon}_{5}=\frac{u}{a_{3}}, \bar{\varepsilon}_{6}=\frac{u}{a_{2}},
$$

where $u$ is the arbitrarily imposed displacement (see Tables 1 and 2). Once the linear elastic problem, characterised by the boundary conditions of Tables 1 and 2, is solved we can get the corresponding stress field whose volume average value for the RVE is computed as follows:

$$
\bar{\sigma}_{\alpha}=\frac{1}{V_{\mathrm{RVE}}} \int_{V_{\mathrm{RVE}}} \sigma_{\alpha}\left(x_{1}, x_{2}, x_{3}\right) d V, \quad \alpha=1,2, \cdots, 6 .
$$

Through the firsts three static analyses we are able to determine the components of the firsts three columns of the stiffness tensor $\mathbf{C}$ while through the last three load cases we can determine the components belonging to the second half of its main diagonal, see Eq. (6). After calculating all nine independent components of $\mathbf{C}$ we can evaluate the effective elastic moduli of the honeycomb core in terms of the stiffness tensor components using the well-known relationships reported in [17].

As a final remark, the equivalent density of the core is evaluated through the following relationship:

$$
\rho_{\mathrm{RVE}}=\frac{\rho t_{c}\left[l_{1}+2 l_{2}+2 t_{c} \tan \left(\frac{\theta}{2}\right)\right]}{\left\{2 l_{2}+t_{c} \tan \left(\frac{\vartheta}{2}\right)+\left[l_{1}+t_{c} \tan \left(\frac{\vartheta}{2}\right)\right] \cos (\vartheta)\right\}\left\{\left[l_{1}+t_{c} \tan \left(\frac{\vartheta}{2}\right)\right] \sin (\vartheta)+t_{c}\right\}} .
$$

where $\rho$ is the density of the material of the cell walls.

\section{Numerical study}

In order to prove the effectiveness of the proposed FE model we performed a numerical study by comparing our results with those obtained using the models of Burton and Noor [11] and Grediac [16].

The sandwich structure considered in this work is made of an aluminium honeycomb core and carbon composite skins, this last being a typical combination of materials employed in some real-world aerospace engineering applications, like those presented in $[8,9,12,25]$. The material properties of the aluminium alloy used for the honeycomb core as well as those of the "elastic air" (these last taken from [2]) are listed in Table 3. We firstly conducted the numerical tests on a reference honeycomb core whose unit cell sizes are given in Table 3 (taken from [1]) while, secondly, we carried out a sensitivity analysis 
(in terms of all the geometric parameters of the unit cell) on the full set of the effective elastic properties.

\subsection{Comparison with existing analytical and numerical models}

The effective material properties for the reference unit cell (having the geometrical dimensions listed in Table 3) obtained using our FE model as well as those evaluated using the approaches of Burton and Noor [11] and Grediac [16] are listed in Table 4. In order to calculate with a good accuracy level the effective material properties of the core a convergence study in terms of mesh size has been carried out. In particular, in Fig. 5 we show the results of the convergence analysis for the equivalent elastic moduli in terms of number of divisions $n_{\text {div }}$ of the mesh along the cell wall thickness $t_{c}$. As it can be seen the convergence is reached when $n_{\text {div }}$ is equal to four: this corresponds to a FE model having a total number of 52009 DOFs. However, when looking at the results of Fig. 5 one can notice that a FE model with only one division along the thickness direction $t_{c}$ is sufficient to properly capture the results, being the maximum relative error of about $4.7 \%$ for the in-plane shear modulus $G_{12}$ which reduces from $0.67 \mathrm{MPa}$ (one division) to $0.64 \mathrm{MPa}$ (four divisions). The main reason underlying this fact is that the average value of the stress components (involved into the evaluation of the equivalent elastic properties, see Eqs. (6)-(8)) is slightly influenced when passing from one to four divisions along $t_{c}$. On the other hand the use of a FE model of the RVE with four elements within the thickness lead us to properly describe the correct variation of the normal as well as the shear stress fields through the thickness (see Figs. 6-8). These considerations led us to use, in the present work, a FE model of the RVE characterised by four divisions along the cell thickness. It is worth noting that the results of Table 4 are compared by considering, in the framework of the models of Burton and Noor and Grediac, a unit cell having the same middle-surface as that of our FE model. As it can be easily seen, the behaviour of the core at the macro-scale is orthotropic with the main orthotropy axes aligned with those of the Cartesian coordinate system of Fig. 2.

For this reference case, where we consider a regular hexagonal honeycomb cell with thin walls, the results given by our 3D solid model globally agree with those found by Burton and Noor and Grediac. In particular, concerning the evaluation of the three out-of-plane moduli $E_{3}, G_{13}$ and $G_{23}$, the relative difference between our model and [11, 16] is very low: $1 \%$ for $G_{23}$ and about $2 \%$ for both $G_{13}$ and $E_{3}$.

On the other hand, if we consider the in-plane moduli $E_{1}, E_{2}, G_{12}$ and the out-of-plane Poisson ratios $\nu_{13}$ and $\nu_{23}$ the results obtained with our 3D FE-based model slightly differ from those provided by $[11,16]$. The relative difference ranges from $8 \%$ for $E_{1}$ to $23.5 \%$ for $G_{12}$. The main reason underlying the previous differences is in the use of shell-based models and theories as done in $[11,16]$ which are not able to properly evaluate the previous quantities. To understand this fact, let us consider the evaluation of the in-plane shear 
modulus $G_{12}$ which is calculated through the following relationship:

$$
G_{12}=C_{66}=\bar{\sigma}_{6} / \bar{\varepsilon}_{6}
$$

From Eq. (10) we can see that $G_{12}$ depends only upon the average stress $\bar{\sigma}_{6}$ and upon the imposed average strain $\bar{\varepsilon}_{6}$. This means that the value of the in-plane shear modulus depends on the accuracy of the numerical evaluation of the in-plane shear stress field of the RVE, which is influenced by the following aspects:

- $\bar{\sigma}_{6}$ depends upon the correct evaluation of the normal and shear stress components in the oblique side $\left(\sigma_{\eta \eta}, \sigma_{\xi \xi}\right.$ and $\left.\sigma_{\eta \xi}\right)$; indeed, all of these stresses are involved in the evaluation of $\bar{\sigma}_{6}$ in the global frame of the RVE $\left\{O ; x_{1}, x_{2}, x_{3}\right\}$, see Figs. 6 and 7 ;

- as shown in Figs. 6 and 7, the previous stress components vary through the thickness of the oblique side and such variation is more pronounced at the intersection between the oblique and the horizontal walls;

- in the oblique face of the unit cell the normal out-of-plane stress $\sigma_{\eta \eta}$ as well as the shear stress $\sigma_{\eta \xi}$ are non-negligible, being the order of magnitude of such components the same as the normal in-plane stress $\sigma_{\xi \xi}$, see Figs. 6 and 7;

- the shear stress $\sigma_{6}$ in the top and bottom horizontal sides of the unit cell varies through the thickness of the wall, as shown in Fig. 8.

The previous aspects cannot be correctly evaluated in the framework of a shell-based model such as those used in $[11,16]$. In particular the out-of-plane normal stress in the oblique wall is null for a shell model and the through-the-thickness variation of the shear stress can be properly evaluated only by higher order shell theories. Similar considerations can be repeated for the calculation of the first three columns of the stiffness tensor of the core and hence for the evaluation of the rest of the elastic properties which depend upon these quantities, see [17].

\subsection{Sensitivity analysis}

Together with the determination of the effective elastic properties of the basic configuration having the geometry of Table 3, we also conducted a sensitivity analysis in which we change, one at a time, every geometric parameter of the unit cell by keeping constant the others. When one of these parameters varies the rest get the values of the reference unit cell as reported in Table 3. For this reason such an analysis can be seen as an analysis of the mechanical response of the RVE, in terms of its effective material properties, in the neighbourhood of the reference configuration. Looking at the results shown in Figs. 9-13 we can deduce the following facts: 
- when varying the parameter $l_{1}$ between $10 t_{c}$ and $100 t_{c}$ (Fig. 9) the trend of the in-plane elastic properties are in agreement with those provided by the model of Burton and Noor. Concerning the out-of-plane Young's modulus $E_{3}$ it is slightly underestimated by our 3D FE model when compared to that given by Burton and Noor and this gap increases when $l_{1}$ decreases. This phenomenon is due to the fact that when $l_{1}$ decreases it becomes of the same order of magnitude as the wall thickness $t_{c}$. Under this condition the mechanical behaviour of the oblique side of the cell is no longer that of a thin plate, thus the model of Burton and Noor overestimates such modulus. Similar considerations could be done both for the shear moduli and the Poisson's ratios;

- when varying the parameter $l_{2}$ between $0.05 l_{1}$ and $0.5 l_{1}$ (Fig. 10) the trend of the results of our model globally agrees with that found using the model of Burton and Noor; the relative difference on the values of the different moduli found using the two models can be explained through the considerations previously done for the reference geometry of the unit cell. This relative difference remains constant for all the elastic properties, with the exception of $G_{12}$ whose relative difference strongly increases when $l_{2}$ decreases: this is due to the fact that when $l_{2}$ becomes of the same order of magnitude as $t_{c}$, both the bottom and top horizontal sides of the unit cell cannot be modelled as thin plates, thus a shell-based model is no longer adapted to correctly capture such a phenomenon;

- when varying the parameter $t_{c}$ between $0.02 l_{2}$ and $0.1 l_{2}$ (Fig. 11) the difference between the results of the present model and those issued from the model of Burton and Noor increases with $t_{c}$. This phenomenon can be easily explained: when the wall thickness increases, the influence of the true 3D geometry of the unit cell on the evaluation of the effective material properties becomes more and more important;

- when varying the parameter $\vartheta$ between $5 \mathrm{deg}$ and $90 \mathrm{deg}$ (Fig. 12) the trend of the results found with our model is practically the same as that provided by the model of Burton and Noor. The relative difference between the values of the effective elastic properties found using the two models, increases when $\vartheta$ decreases, with the exception of $G_{13}$ and $G_{23}$. This is due to the fact that when the corrugation angle of the cell decreases the unit cell becomes more and more "flat". In such a configuration the effect of the out-of-plane stresses $\bar{\sigma}_{2}$ and $\bar{\sigma}_{6}$ on the calculation of the effective core properties plays a crucial role, thus a shell-like model is not sufficient to properly capture these phenomena;

- when varying the parameter $h_{c}$ between $l_{1}$ and $10 l_{1}$ (Fig. 13) we show that, actually, the assumption underlying the model of Burton and Noor that the core properties 
do not vary with the core height is correct for seven elastic properties $\left(E_{1}, E_{2}\right.$, $E_{3}, G_{12}, \nu_{12}, \nu_{23}$ and $\left.\nu_{13}\right)$. On the contrary, according to the model of Grediac [16], this assumption cannot be accepted when evaluating $G_{13}$ that varies with the parameter $h_{c}$. Nevertheless, unlike the results provided by Grediac our model is able to capture also the weak variation of $G_{23}$ with the core thickness. Moreover, the relative difference calculated on $G_{23}$ decreases when $h_{c}$ increases, ranging from $1 \%$ to $0,1 \%$.

To be remarked that when varying each geometrical parameter of the cell, the curve describing the variation of the out-of-plane shear modulus $G_{13}$ always lies between the lower (LB) and upper (UB) bounds given by the model of Burton and Noor which are calculated using the strain energy associated firstly with a stress distribution satisfying the equilibrium (for the LB) and secondly with a strain field allowing compatible deformation (for the UB). The reader is addressed to [11] for a deeper insight in the matter. As a conclusive remark of this section, we want to highlight the importance of using solid elements to build the FE model of the repetitive unit cell which are able to properly capture the influence of the true 3D stress field on the evaluation of the effective elastic properties of the honeycomb core.

\section{Conclusions}

The main aim of the present work is to deal with the problem of the optimum design of a sandwich panel composed of two laminated skins and a honeycomb core. In this first part of the work we presented the numerical homogenisation technique as well as the related 3D FE model of the unit cell that will be used within the first level of the optimisation procedure (see Part II). In particular, we need an adequate model of the honeycomb core, at the meso-scale, able to properly predict its equivalent material properties (at the macroscale) for any combination of design variables that, in the framework of the optimisation procedure, could give rise to a geometry of the unit cell which is far away from a classical shell-like geometry (i.e. a cell with thin walls).

To these purposes, firstly we demonstrated that shell-based models cannot correctly represent the true geometry of the unit cell of the honeycomb core and, consequently, they are not able to properly capture the influence of the real 3D stress field on the determination of the effective core properties. For these reasons, in this paper, we used a 3D solid FEbased model of the repetitive unit cell of the honeycomb core to predict its effective material properties in the most general case, i.e. by taking into account any combination of the geometric parameters of the unit cell.

The material properties determined using our model were, in addition, compared with those obtained using the models of Burton and Noor [11] and Grediac [16]. Numerical 
results show that, for a hexagonal unit cell with thin walls, the out-of-plane elastic moduli found by our model globally agree with those provided by shell-based analytical and numerical models. On the contrary, the in-plane elastic moduli found using our model show a relative difference ranging from $8 \%$ to $23.5 \%$ (depending on the considered quantity) with respect to those provided by shell-based models. The main reason underlying such differences is the influence of the "local" out-of plane normal stress over the cell walls which is identically null in the framework of a shell-based model. Moreover, through an analysis on the local stress field within the cell walls along with a sensitivity analysis, we proved that such models are no longer appropriate in the framework of an optimisation procedure that aims to take into account, among the design variables, the full set of the geometric parameters characterising the unit cell. In fact, for those configurations in which the thickness-to-side ratio of the cell walls goes beyond the limits imposed by a shell-like model, a 3D solid model of the unit cell is therefore necessary to properly describe the effect of the 3D stress field on the evaluation of the full set of material properties of the equivalent solid that will be used at the macro-scale.

Part II of the present work will cover the formulation of the optimisation problem and provide a detailed description of the two-level optimisation strategy; numerical examples will be presented in order to demonstrate the effectiveness of the proposed approach.

\section{References}

[1] HexWeb honeycomb attributes and properties. Duxford, Cambridge, UK, 1999.

[2] ANSYS, Inc., 275 Technology Drive, Canonsburg, PA 15317. ANSYS Mechanical APDL Modeling and Meshing Guide, 2012.

[3] ASTM, West Conshohocken, Pa. C273 standard method of shear test in flatwise plane of flat sandwich constructions or sandwich cores, 1970.

[4] ASTM, West Conshohocken, Pa. C271 standard test method for density of core materials for structural sandwich contructions, 1988.

[5] ASTM, West Conshohocken, Pa. C365 standard test methods for flatwise compressive strangth of sandwich cores, 1988.

[6] ASTM, West Conshohocken, Pa. D6790 standard test method for determining Poisson's ratio of honeycomb cores, 2002.

[7] E. J. Barbero. Finite element analysis of composite materials. Taylor and Francis Group, 2008. 
[8] T. Besant, G.A.O. Davies, and D. Hitchings. Finite element modelling of low velocity impact of composite sandwich panels. Composites Part A: Applied science and manufacturing, 32:1189-1196, 2001.

[9] B. L. Buitrago, C. Santiuste, S. Sánchez-Sáez, E. Barbero, and C. Navarro. Modelling of composite sandwich structures with honeycomb core subjected to high-velocity impact. Composite Structures, 92:2090-2096, 2010.

[10] V. N. Burlayenko and T. Sadowski. Effective elastic properties of foam-filled honeycomb cores of sandwich panels. Composite Structures, 92:2890-2900, 2010.

[11] W. S. Burton and A. K. Noor. Assessment of continuum models for sandwich panel honeycomb cores. Computer Methods in Applied Mechanics and Engineering, 145:341$360,1997$.

[12] K. Diamanti, C. Soutis, and J.M. Hodgkinson. Lamb waves for the non-destructive inspection of monolithic and sandwich composite beams. Composites Part A: Applied science and manufacturing, 36:189-195, 2005.

[13] C. C. Foo, G. B. Chai, and L. K. Seah. Mechanical properties of nomex material and nomex honeycomb structure. Composite Structures, 80:588-594, 2007.

[14] X. Frank Xu and P. Qiao. Homogenized elastic properties of honeycomb sandwich with skin effect. International Journal of Solids and Structures, 39:2153-2188, 2002.

[15] L. J. Gibson and M. F. Ashby. Cellular solids - Structure and properties. Cambridge University Press, 1997.

[16] M. Grediac. A finite element study of the transverse shear in honeycomb cores. International Journal of Solids and Structures, 30(13):1777-1788, 1993.

[17] R. M. Jones. Mechanics of composite materials. McGraw-Hill, 1975.

[18] A. Karakoç and J. Freund. Experimental studies on mechanical properties of cellular structures using nomex honeycomb cores. Composite Structures, 94:2017-2024, 2012.

[19] F. Meraghni, F. Desrumaux, and M. L. Benzeggagh. Mechanical behaviour of cellular core for structural sandwich panels. Composites: Part A, 30:767-779, 1999.

[20] M. Montemurro, A. Vincenti, and P. Vannucci. A two-level procedure for the global optimum design of composite modular structures - application to the design of an aircraft wing. Part 1: theoretical formulation. Journal of Optimization Theory and Applications, 155(1):1-23, 2012. 
[21] M. Montemurro, A. Vincenti, and P. Vannucci. A two-level procedure for the global optimum design of composite modular structures - application to the design of an aircraft wing. Part 2: numerical aspects and examples. Journal of Optimization Theory and Applications, 155(1):24-53, 2012.

[22] T. Sadowski and J. Bȩc. Effective properties for sandwich plates with aluminium foil honeycomb core and polymer foam filling - static and dynamic response. Computational Materials Science, 50:1269-1275, 2011.

[23] C. W. Schwingshackl, G. S. Aglietti, and P. R. Cunningham. Determination of honeycomb material properties: existing theories and an alternative dynamic approach. Journal of Aerospace Engineering, 19(3):177-183, 2006.

[24] G. Shi and P. Tong. Equivalent transverse shear stiffness of honeycomb cores. International Journal of Solids and Structures, 32(10):1383-1393, 1995.

[25] E. A. Taylor, M. K. Herbert, B. A. M. Vaughan, and J. A. M. McDonnell. Hypervelocity impact on carbon fibre reinforced plastic / aluminium honeycomb: comparison with whipple bumper shields. International Journal of Impact Engineering, 23:883893, 1999.

[26] P. Vannucci. Plane anisotropy by the polar method. Meccanica, 40:437-454, 2005.

[27] A. Vautrin. Mechanics of sandwich structures. Springer, 2010.

[28] G. Verchery. Les invariants des tenseurs d'ordre 4 du type de l'élasticité. VIllard-deLans, (France), 1979. Proc. of colloque Euromech 115.

[29] J. R. Vinson. The behavior of sandwich structures of isotropic and composite materials. Technomic Publishing Company, 1999.

[30] J. Zhang and M. Ashby. The out-of-plane properties of honeycombs. International Juornal of Mechanical Science, 145:341-360, 1997. 


\section{Tables}

\begin{tabular}{|lccc|lccc|lcc|cc|}
\hline $1^{\text {st }}$ load case & & & \multicolumn{3}{l|}{$2^{\text {nd }}$ load case } & & & $3^{\text {rd }}$ load case \\
\hline Nodes & $U_{1}$ & $U_{2}$ & $U_{3}$ & Nodes & $U_{1}$ & $U_{2}$ & $U_{3}$ & Nodes & $U_{1}$ & $U_{2}$ & $U_{3}$ \\
\hline$x_{1}=0$ & 0 & free & free & $x_{1}=0$ & 0 & free & free & $x_{1}=0$ & 0 & free & free \\
$x_{1}=a_{1}$ & $u$ & free & free & $x_{1}=a_{1}$ & 0 & free & free & $x_{1}=a_{1}$ & 0 & free & free \\
$x_{2}=0$ & free & 0 & free & $x_{2}=0$ & free & 0 & free & $x_{2}=0$ & free & 0 & free \\
$x_{2}=a_{2}$ & free & 0 & free & $x_{2}=a_{2}$ & free & $u$ & free & $x_{2}=a_{2}$ & free & 0 & free \\
$x_{3}=0$ & free & free & 0 & $x_{3}=0$ & free & free & 0 & $x_{3}=0$ & free & free & 0 \\
$x_{3}=a_{3}$ & free & free & 0 & $x_{3}=a_{3}$ & free & free & 0 & $x_{3}=a_{3}$ & free & free & $u$ \\
\hline
\end{tabular}

Table 1: Boundary conditions for the FE model of the RVE: $1^{\text {st }}, 2^{\text {nd }}$ and $3^{\text {rd }}$ static analyses.

\begin{tabular}{|lccc|lcccc|lcccc|}
\hline $4^{\text {th }}$ load case & & & \multicolumn{3}{l|}{$5^{\text {th }}$ load case } & & \multicolumn{3}{l|}{$6^{\text {th }}$ load case } \\
\hline Nodes & $U_{1}$ & $U_{2}$ & $U_{3}$ & Nodes & $U_{1}$ & $U_{2}$ & $U_{3}$ & Nodes & $U_{1}$ & $U_{2}$ & $U_{3} \mid$ \\
\hline$x_{1}=0$ & 0 & free & free & $x_{1}=0$ & free & 0 & 0 & $x_{1}=0$ & free & 0 & 0 \\
$x_{1}=a_{1}$ & 0 & free & free & $x_{1}=a_{1}$ & free & 0 & 0 & $x_{1}=a_{1}$ & free & 0 & 0 \\
$x_{2}=0$ & 0 & free & 0 & $x_{2}=0$ & free & 0 & free & $x_{2}=0$ & 0 & free & 0 \\
$x_{2}=a_{2}$ & 0 & free & 0 & $x_{2}=a_{2}$ & free & 0 & free & $x_{2}=a_{2}$ & $u$ & 0 & 0 \\
$x_{3}=0$ & 0 & 0 & free & $x_{3}=0$ & 0 & 0 & free & $x_{3}=0$ & free & free & 0 \\
$x_{3}=a_{3}$ & 0 & $u$ & 0 & $x_{3}=a_{3}$ & $u$ & 0 & 0 & $x_{3}=a_{3}$ & free & free & 0 \\
\hline
\end{tabular}

Table 2: Boundary conditions for the FE model of the RVE: $4^{\text {th }}, 5^{\text {th }}$ and $6^{\text {th }}$ static analyses.

\begin{tabular}{|c|c|c|c|c|}
\hline \multicolumn{5}{|c|}{ Material properties } \\
\hline Aluminiu & & $\begin{array}{c}E[\mathrm{MPa}] \\
70000\end{array}$ & $\begin{array}{c}\nu \\
0.33\end{array}$ & $\begin{array}{c}\rho\left[\mathrm{Kg} / \mathrm{mm}^{3}\right] \\
2.7 \times 10^{-6}\end{array}$ \\
\hline Elastic ai & & $\begin{array}{c}E_{\text {air }}[\mathrm{MPa}] \\
1 \times 10^{-3} \\
\end{array}$ & $\begin{array}{c}\nu_{\text {air }} \\
0.0 \\
\end{array}$ & \\
\hline \multicolumn{5}{|c|}{ Geometrical parameters (reference values) } \\
\hline $\begin{array}{c}l_{1}[\mathrm{~mm}] \\
3.666\end{array}$ & $\begin{array}{c}l_{2}[\mathrm{~mm}] \\
1.833\end{array}$ & $\begin{array}{c}t_{c}[\mathrm{~mm}] \\
0.0635\end{array}$ & $\begin{array}{c}\theta[\mathrm{deg}] \\
60\end{array}$ & $\begin{array}{c}h_{c}[\mathrm{~mm}] \\
20\end{array}$ \\
\hline
\end{tabular}

Table 3: Material and geometrical parameters of the unit cell. 


\begin{tabular}{l|l|l|l}
\hline Properties & Present & Burton \& Nooor [11] & Grediac [16] \\
\hline$E_{1}[\mathrm{MPa}]$ & 0.884 & 0.815 & 0.815 \\
$E_{2}[\mathrm{MPa}]$ & 0.918 & 0.815 & 0.815 \\
$E_{3}[\mathrm{MPa}]$ & 1812.299 & 1848.185 & 1848.185 \\
$G_{12}[\mathrm{MPa}]$ & 0.640 & 0.489 & 0.489 \\
$G_{23}[\mathrm{MPa}]$ & 262.981 & 260.552 & 260.552 \\
$G_{13}[\mathrm{MPa}]$ & 390.833 & $156.331(\mathrm{LB})$ & 397.088 \\
& & $434.254(\mathrm{UB})$ & \\
$\nu_{12}$ & 0.980 & 1.000 & 1.000 \\
$\nu_{23}$ & $0.161 \times 10^{-3}$ & $0.145 \times 10^{-3}$ & $0.145 \times 10^{-3}$ \\
$\nu_{13}\left[\mathrm{Kg} / \mathrm{mm}^{3}\right]$ & $0.167 \times 10^{-3}$ & $0.145 \times 10^{-3}$ & $0.145 \times 10^{-3}$ \\
$\rho_{\mathrm{RVE}}$ & $6.990 \times 10^{-8}$ & $7.117 \times 10^{-8}$ & \\
\hline
\end{tabular}

Table 4: Effective material properties of the core for the reference geometry of the RVE. 


\section{Figures}
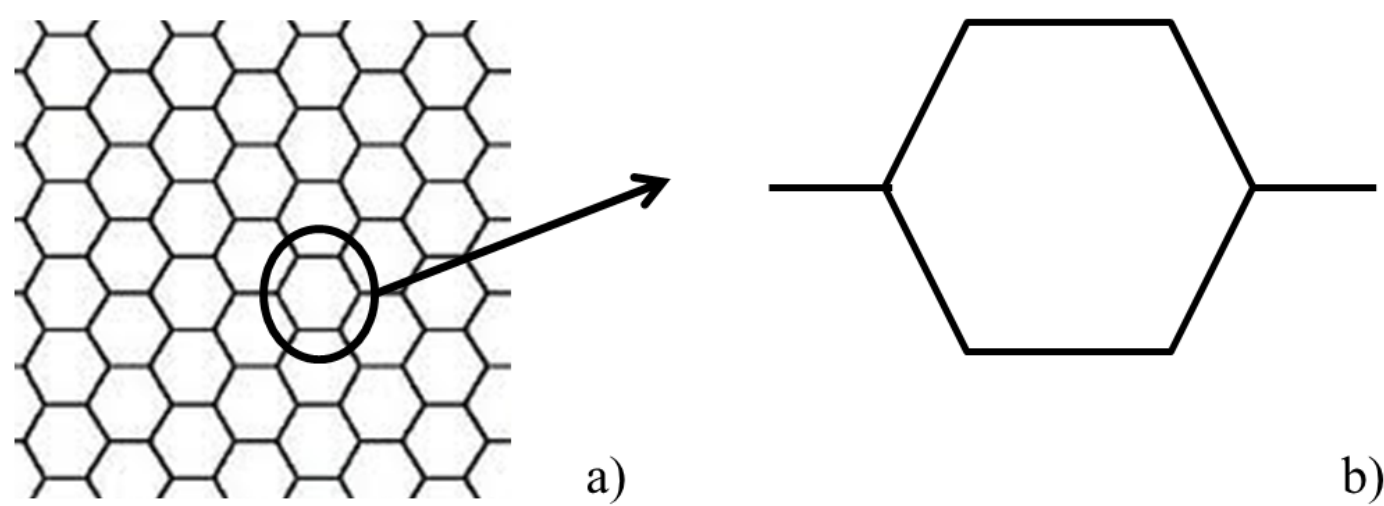

a)

b)

Figure 1: Honeycomb core structure (a) and the repetitive unit cell (b).
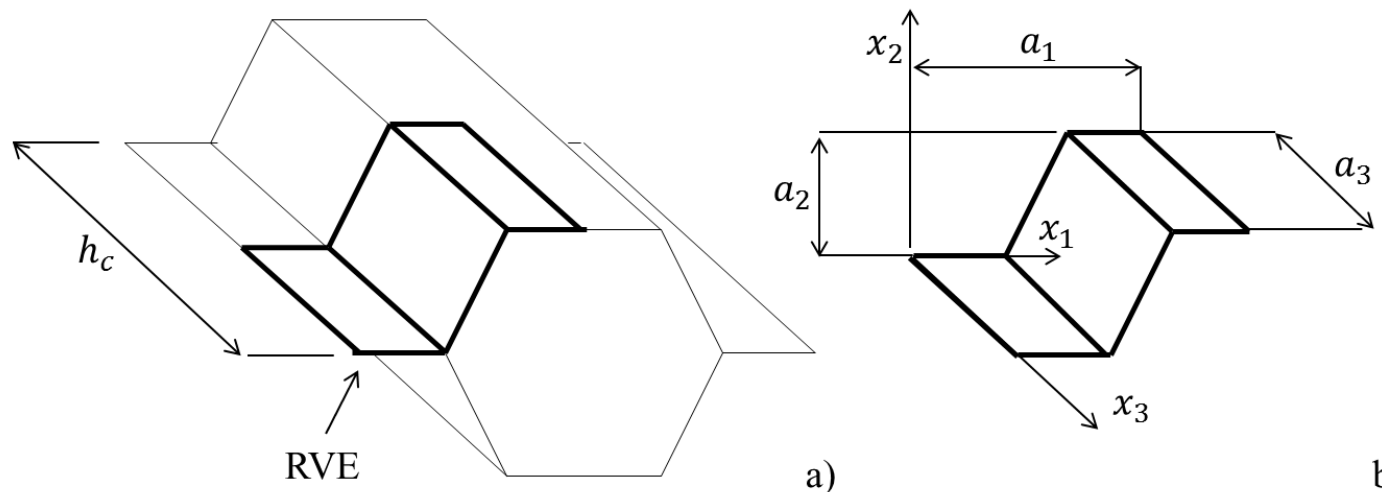

a)

b)

Figure 2: The repetitive unit cell (a) and the overall sizes of the related RVE (b). 


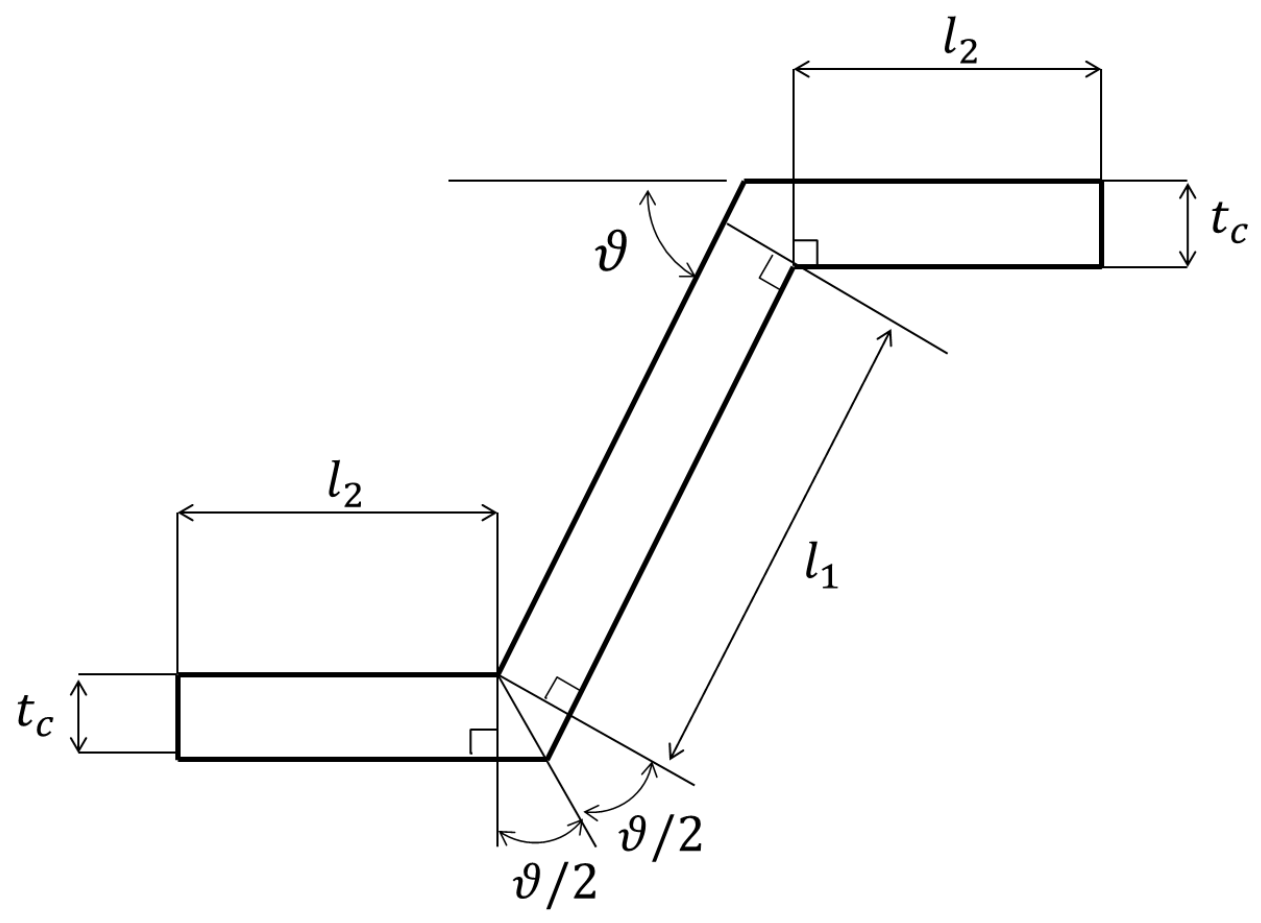

Figure 3: Geometrical parameters of the unit cell.

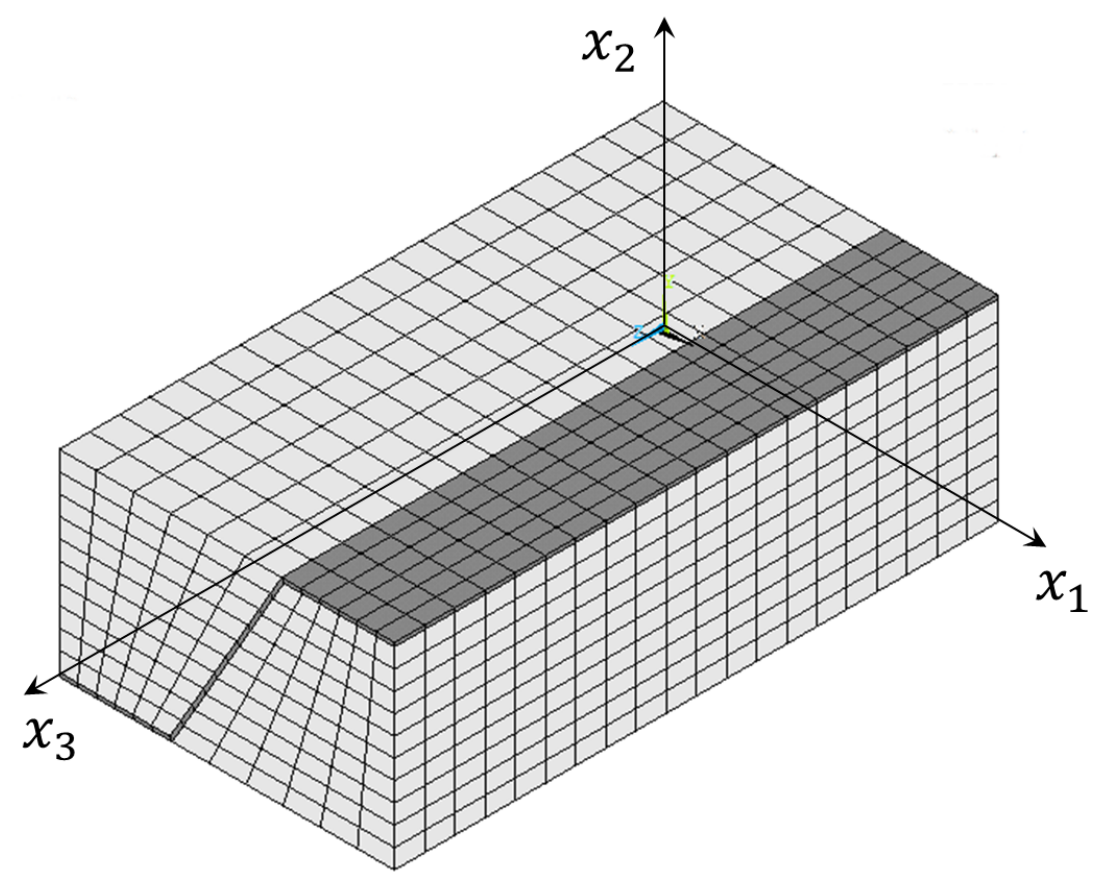

Figure 4: FE model of the RVE. 

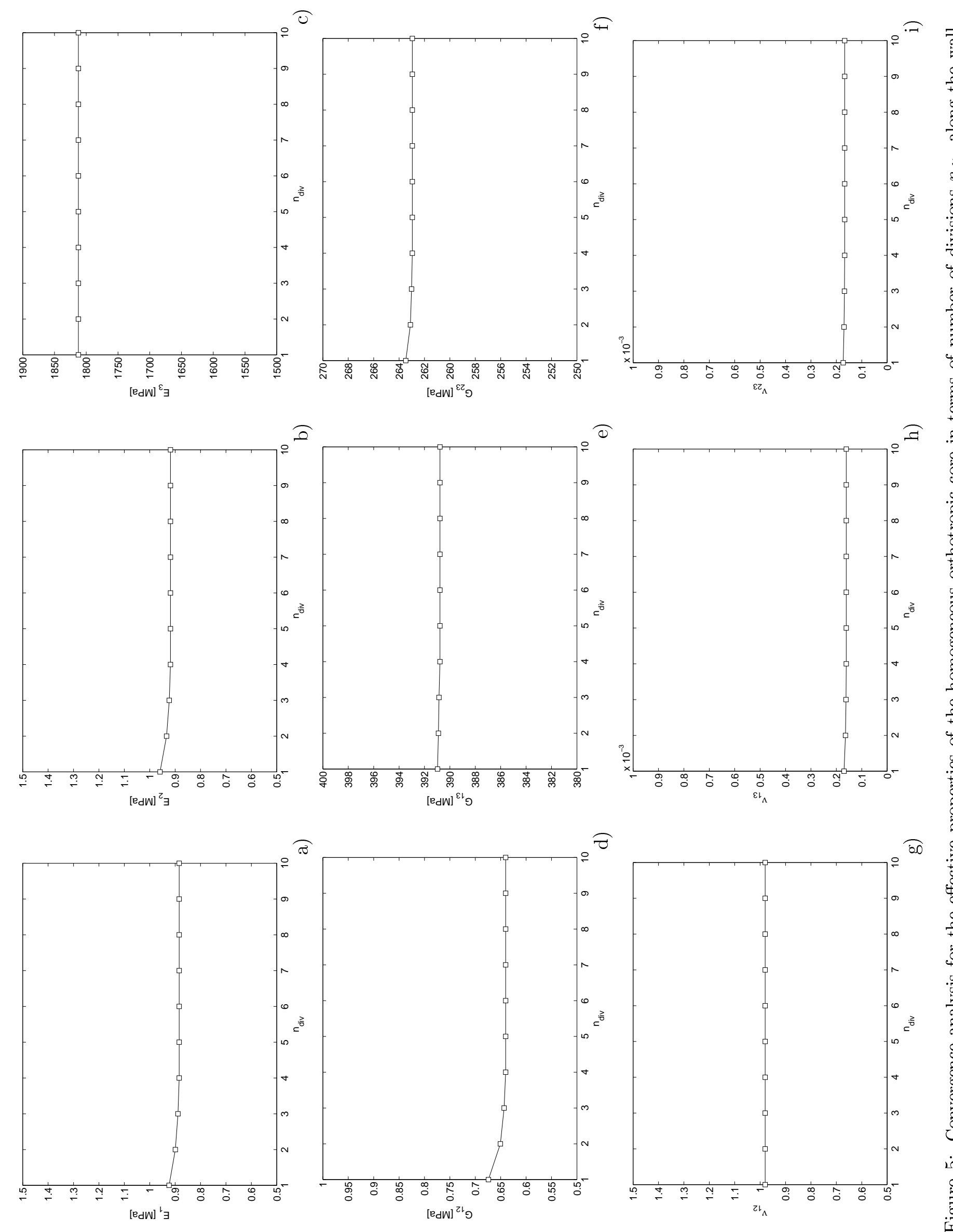

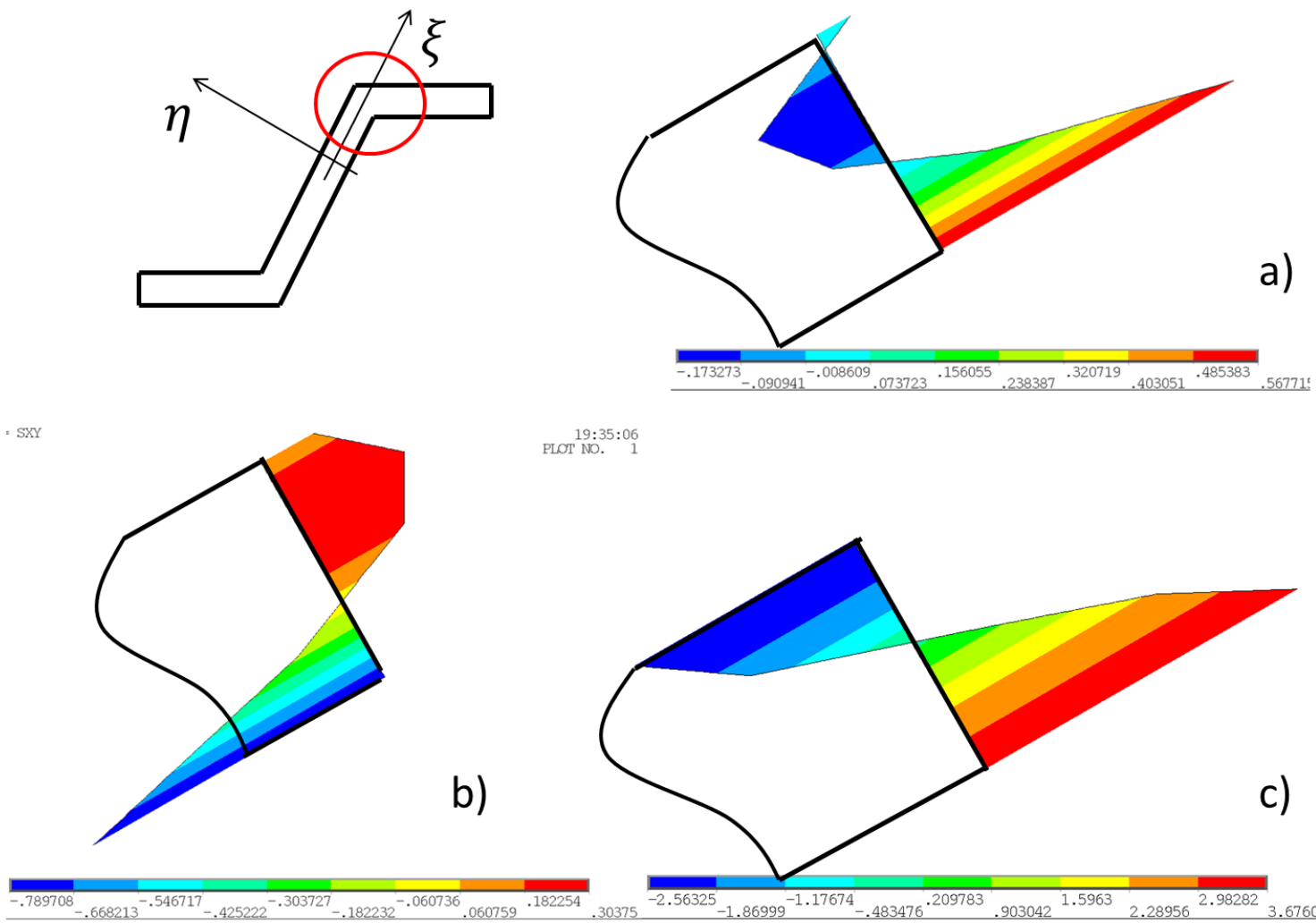

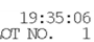
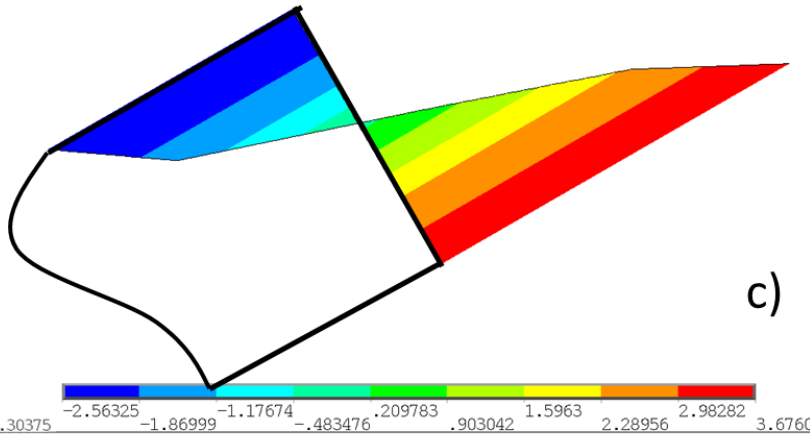

Figure 6: Variation of $\sigma_{\eta \eta}(\eta)(\mathrm{a}), \sigma_{\eta \xi}(\eta)(\mathrm{b})$ and $\sigma_{\xi \xi}(\eta)(\mathrm{c})[\mathrm{MPa}$ ] within the oblique wall of the cell at the intersection with the top horizontal side, $6^{\text {th }}$ load case. 

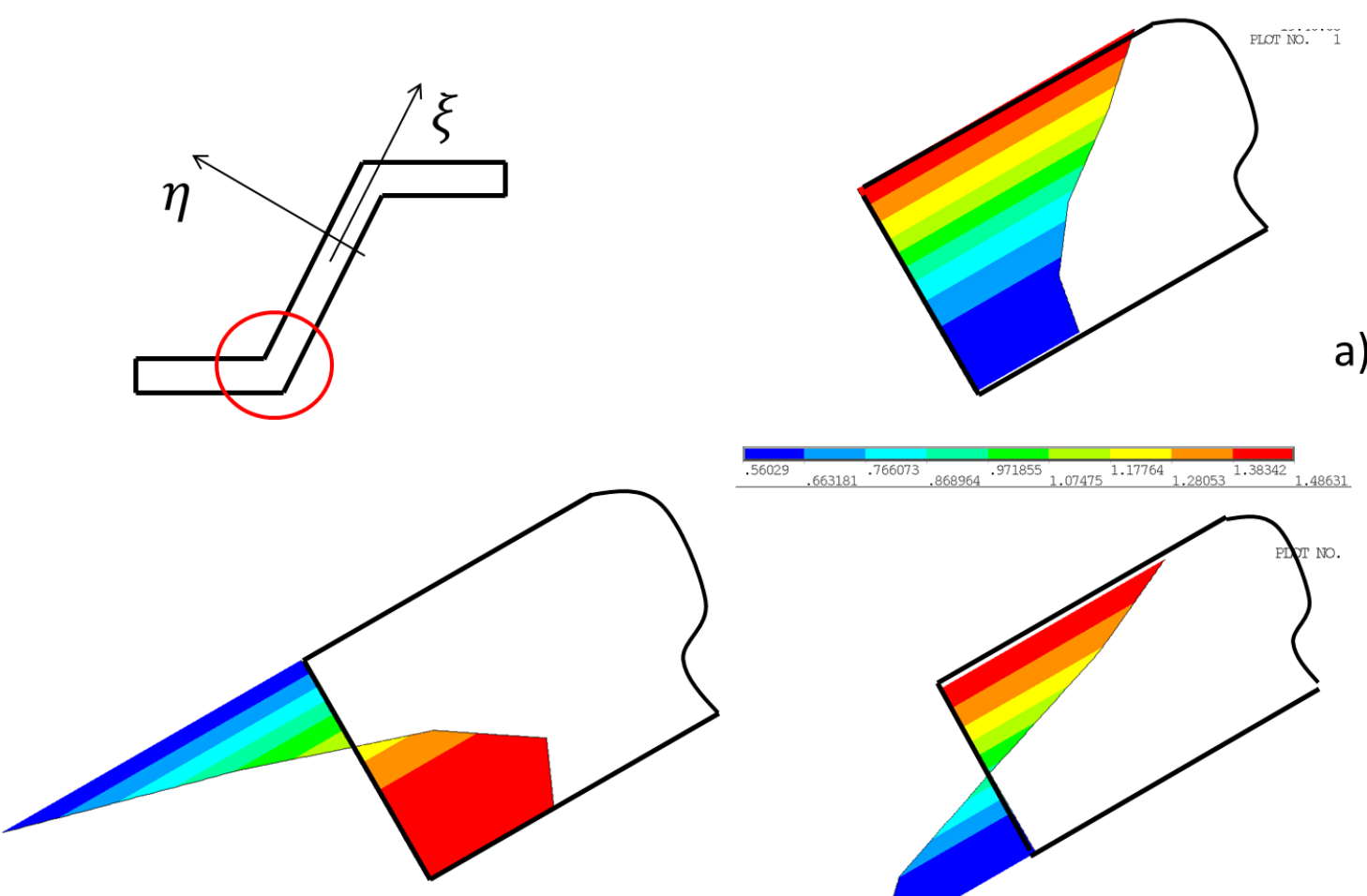

b)

c)
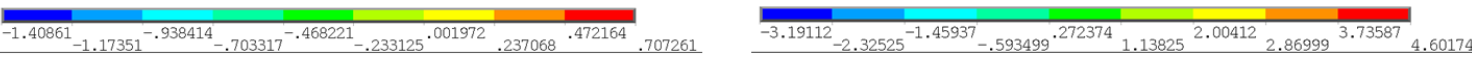

Figure 7: Variation of $\sigma_{\eta \eta}(\eta)(\mathrm{a}), \sigma_{\eta \xi}(\eta)$ (b) and $\sigma_{\xi \xi}(\eta)(\mathrm{c})[\mathrm{MPa}$ within the oblique wall of the cell at the intersection with the bottom horizontal side, $6^{\text {th }}$ load case. 

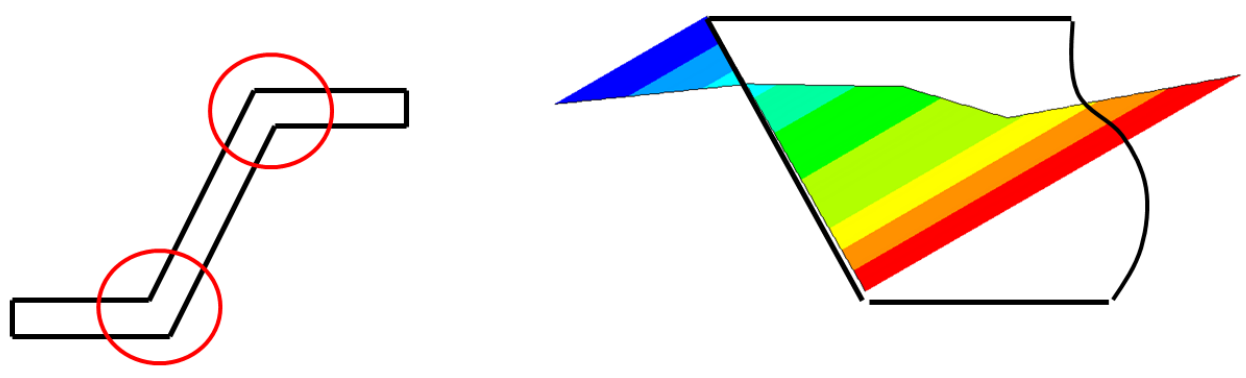

a)

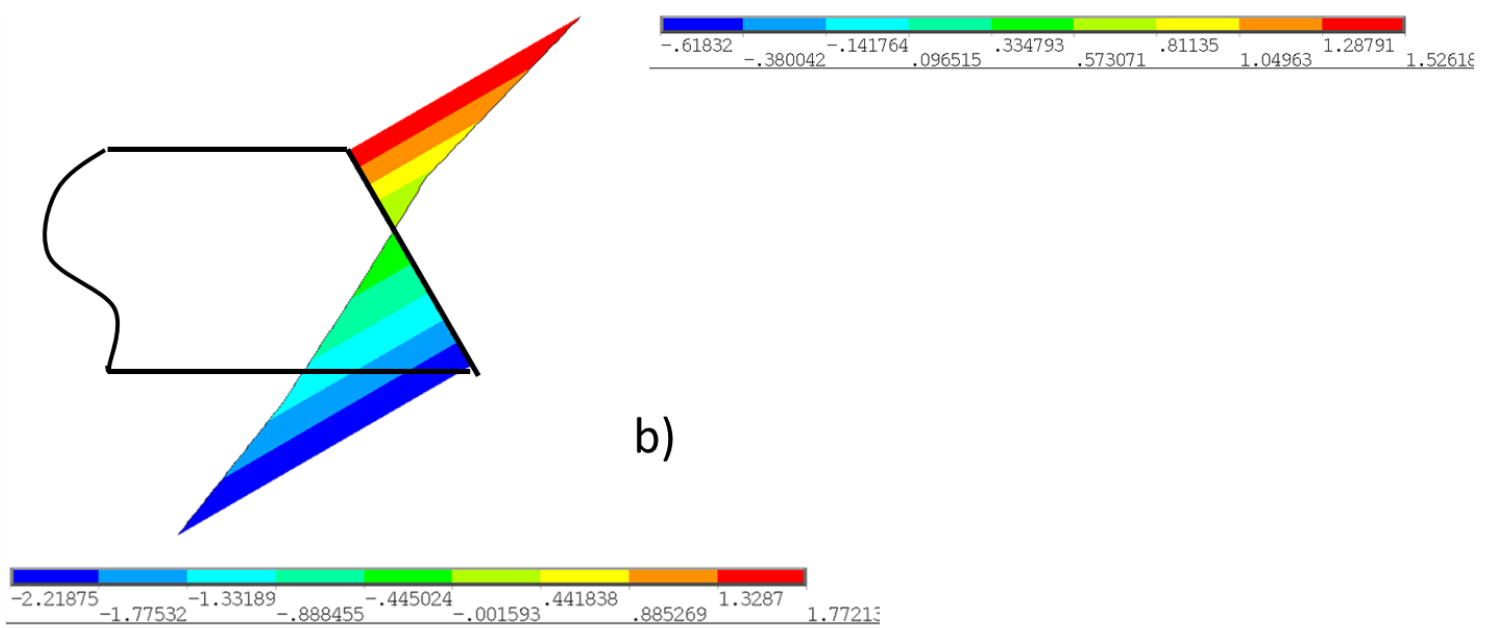

Figure 8: Variation of $\sigma_{6}(\eta)$ [MPa] within the top horizontal wall (a) and the bottom horizontal wall (b) of the cell at the intersection with the oblique side, $6^{\text {th }}$ load case. 

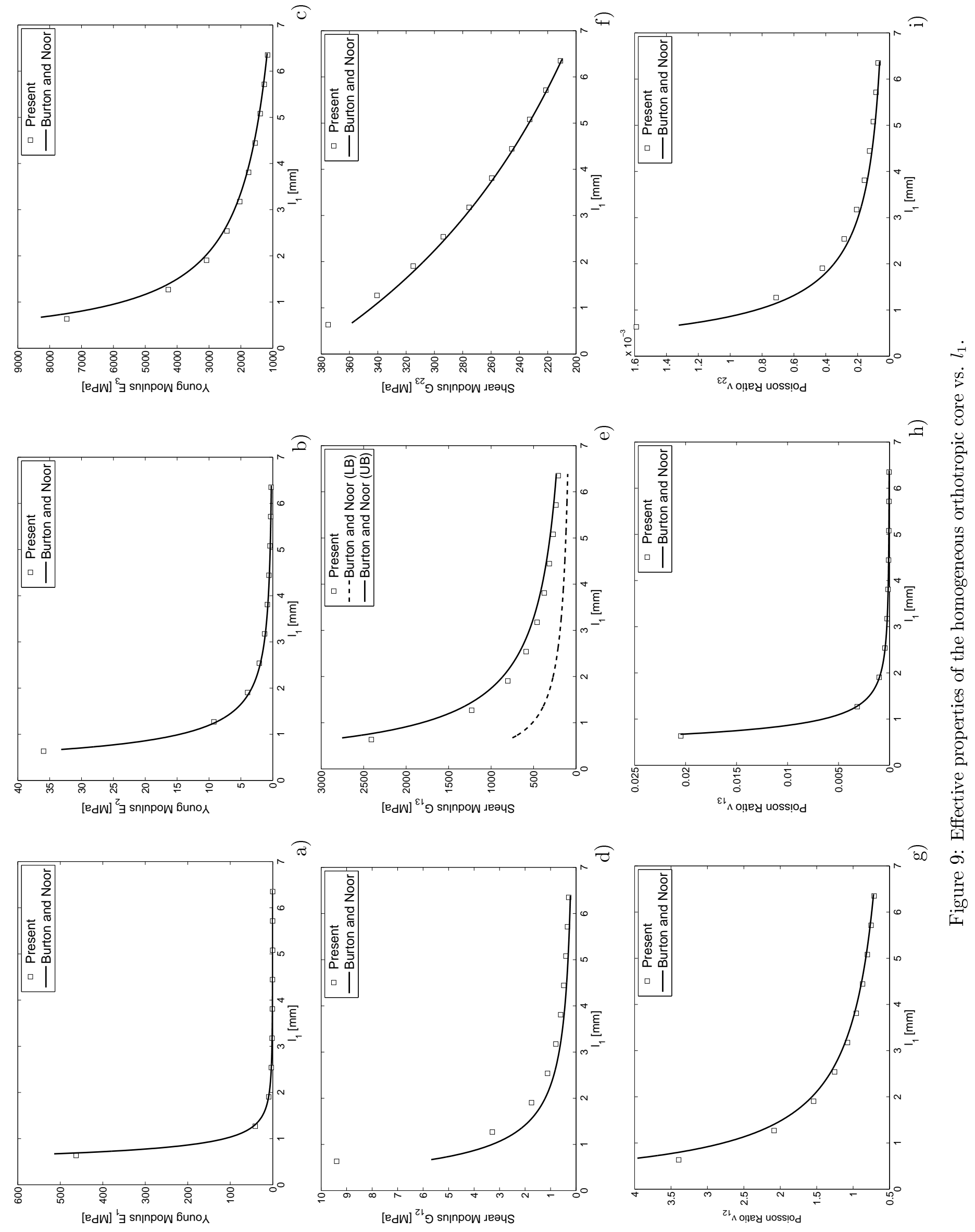

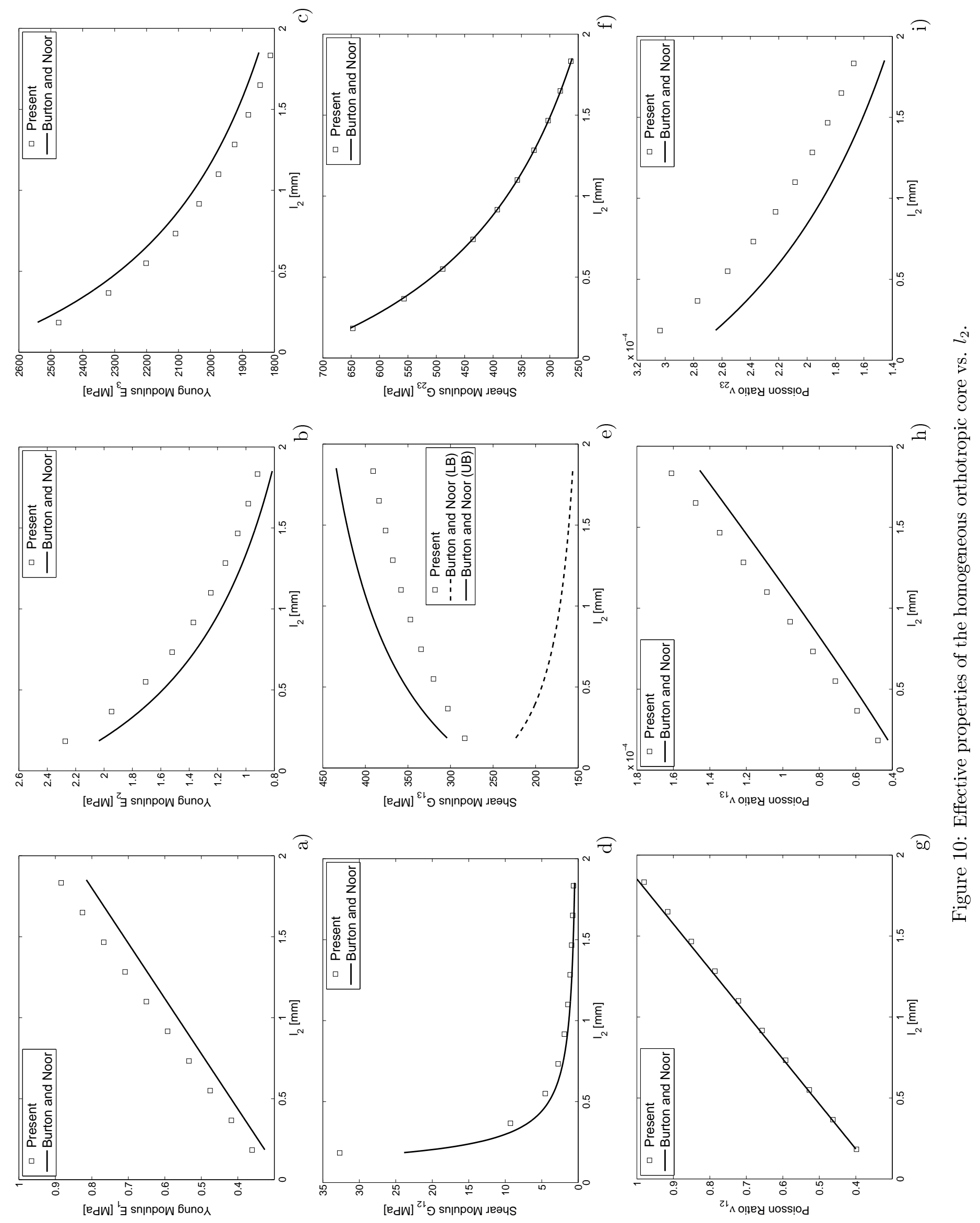

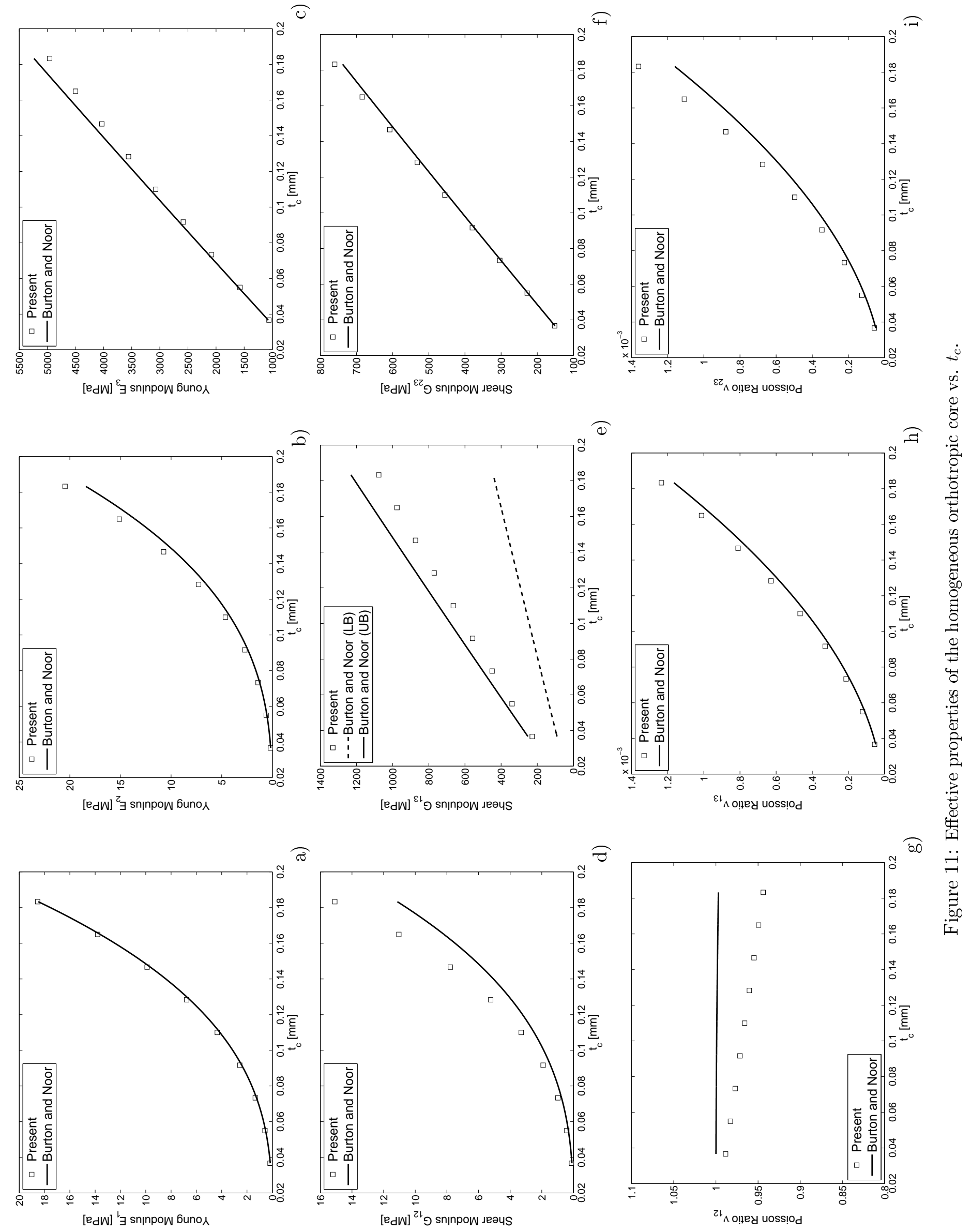

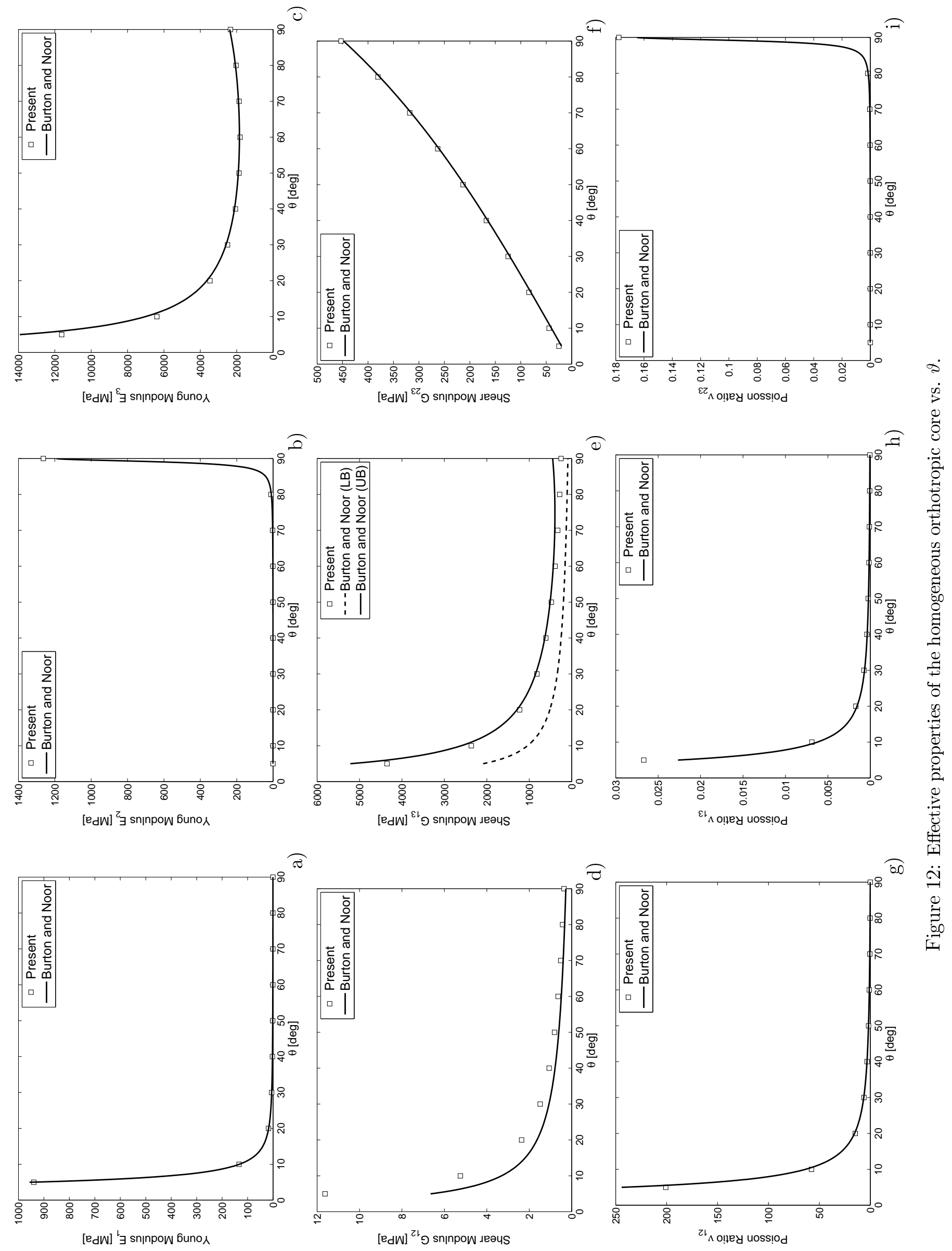

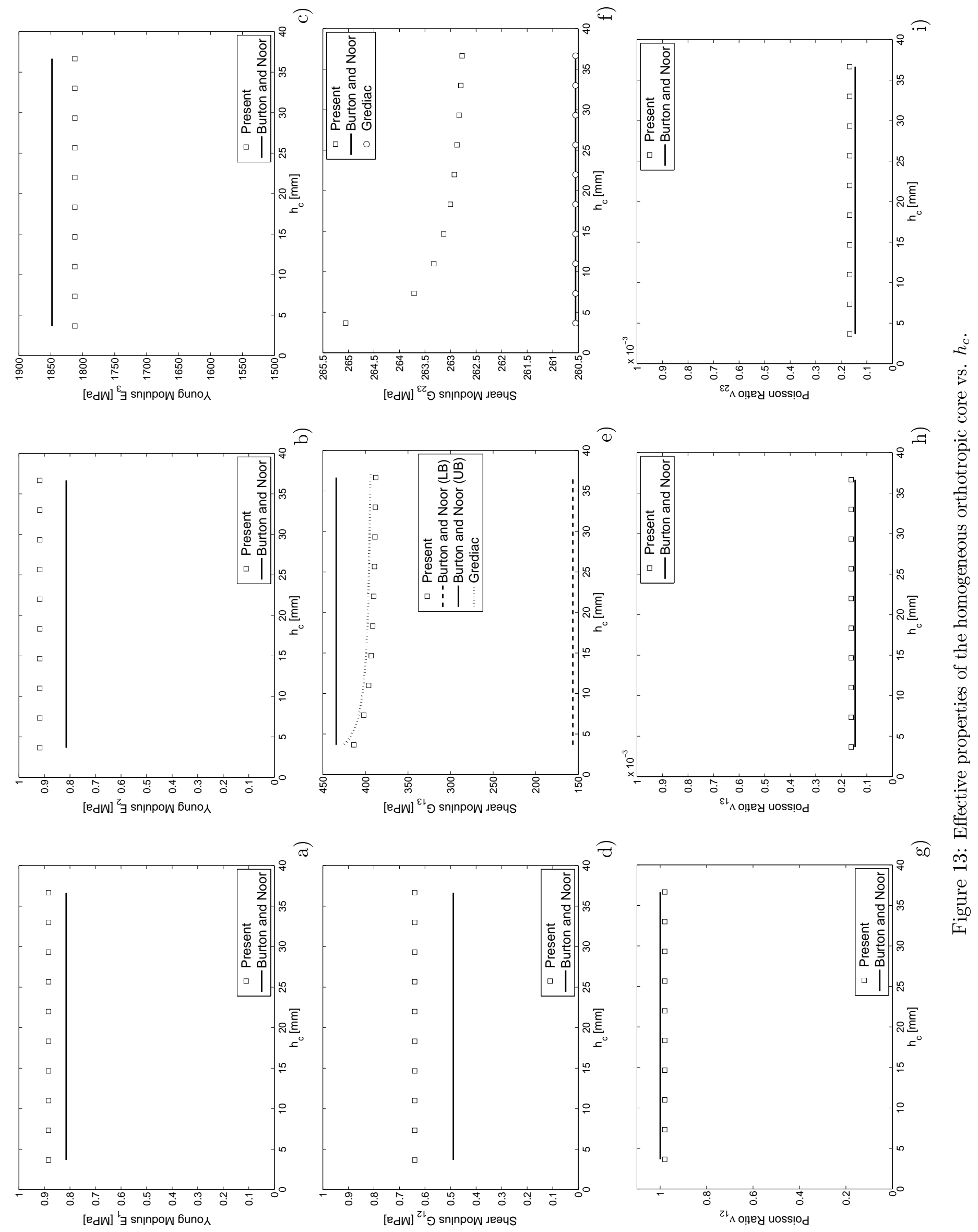